\title{
Prostate Cancer: Cases of Rare Presentation and Rare Metastasis
}

\author{
Hiren Mandaliya ${ }^{a} \quad J$ oshua Sung ${ }^{b} \quad$ Joanna Hill ${ }^{c}$ Ramin Samali ${ }^{a}$ \\ Mathew George ${ }^{d}$ \\ ${ }^{a}$ Tamworth Rural Referral Hospital, ${ }^{b}$ University of New England, Tamworth Rural Referral \\ Hospital, 'University of Newcastle, Tamworth Rural Referral Hospital, 'Medical Oncology, \\ Tamworth Rural Referral Hospital, Tamworth, N.S.W., Australia
}

\section{Key Words}

Prostate cancer $\cdot$ Rare presentation $\cdot$ Metastasis

\begin{abstract}
Prostate cancer is the second most common cancer in men. Diagnosis of early disease is based on prostate biopsy which is carried out because of symptoms of prostatism or asymptomatic rise in PSA. On the other side, advanced disease can locally invade and metastasise to lymph nodes, bones, lungs, etc. Initial presentation of prostate cancer in form of brain metastasis is extremely seldom. Similarly, prostate cancer, which metastasised to the breast, is very rare too. Here, we discuss two unique cases of prostate cancer, one of them had an initial presentation of brain metastasis from prostate adenocarcinoma and the other case had an established diagnosis of prostate cancer metastasised to the breast. In theory, cancer can cause metastatic spread to any part of the body; however diversity into such presentation or progression from prostate cancer has not been frequently noticed.
\end{abstract}

(C) 2015 The Author(s)

Published by S. Karger AG, Basel

\section{Introduction}

Brain metastasis from prostate cancer is a rare and late manifestation. This develops in patients with prolonged survival. In addition, only few cases with brain metastasis preceding the diagnosis of prostate cancer have been reported in literature [1]. Unlike melanoma and carcinomas of the lung, breast, kidney and colon, it is rare for prostate cancer to metastasise to the central nervous system and even rarer to have diagnostic manifestation as a brain metastasis, making it the topic of this case report. 
Mandaliya et al.: Prostate Cancer: Cases of Rare Presentation and Rare Metastasis

Metastasis to the breast from a primary prostate adenocarcinoma is a rare occurrence, as the breast is an uncommon site of metastases in general, and even less frequent in prostate cancer. There is evidence that suggests that hormone manipulation in treatment of prostate cancer could predispose to breast metastasis. Oestrogen therapy, which was previously the mainstay in the treatment of prostate cancer, has been linked to the development of breast metastasis [2].

\section{Case Presentation}

Case 1

The case of a 61-year-old male with a gradual onset right-sided facial palsy, dysarthria and hemiparesis for a few weeks is presented. His past medical history was not significant and did not have a history of smoking or alcohol intake. He was generally well otherwise. His initial CT scan of the brain was suggestive of a left temporal lobe mass, which was confirmed on brain MRI as a large irregularly enhancing mass lesion in the left temporal lobe with solid and cystic components, possible were a primary or metastatic carcinomatous process. His initial blood tests including full blood counts, liver functions, renal functions, electrolytes were normal. However, is tumour markers particularly PSA (prostate specific antigen) was $1,152 \mathrm{mg} / \mathrm{l}$ (normal value less than $5 \mathrm{mg} / \mathrm{l}$ ). He underwent stereotactic craniotomy for excision of the mass. Histopathology showed metastatic adenocarcinoma with immunohistochemistry (CK7 negative, CK20 negative and PSA positive) which favoured metastatic prostatic adenocarcinoma. Post-operatively his PSA dropped to $23 \mathrm{mg} / \mathrm{l}$. CT scan of the chest/abdomen/pelvis did not show any metastatic lesions or prostatic enlargement. However, his bone scan showed increased uptake in multiple ribs and anterior superior iliac spine. He improved remarkably following surgery on neurological aspects. With a diagnosis of metastatic prostatic adenocarcinoma, he underwent palliative whole brain radiotherapy after surgery. He was also commenced on goserelin acetate as an androgen deprivation therapy. His PSA normalised to $3.7 \mathrm{mg} / \mathrm{l}$ within 7 weeks. He has been doing exceptionally well since then and having ongoing clinical biochemical and radiological response without any evidence of disease recurrence nearly 36 months following the initial event.

Case 2

An 80-year-old male was diagnosed with prostate cancer in 2011. It was an adenocarcinoma with Gleeson score of $4+3=7$. His initial PSA was $6.7 \mathrm{mg} / \mathrm{l}$. He was started on leuprorelin and bicalutamide. He has shown ongoing response to dual androgen blockade for nearly 4 years. Early in the same year, he presented with a painless swelling in his right breast. It was a $5 \times 4 \mathrm{~cm}$ sized, hard, non-tender swelling at right breast, without any underlying bone tenderness or lymphadenopathy. Given his long-standing androgen deprivation therapy, the initial diagnosis was gynaecomastia. Ultrasound of the right breast revealed a lobulated retro-areolar lesion of the breast measuring $18 \times 20 \times 46 \mathrm{~mm}$ in diameter with well-defined margins, more in favour of atypical gynaecomastia. There was no abnormality on the right breast and no evidence of ipsilateral axillary or supraclavicular or neck lymphadenopathy. Core biopsy showed a malignant infiltrate with positive staining for PSA. The stains for CK7/CK20, oestrogen receptor, progesterone receptor, CD31, CD34, design and smooth muscle actin were negative. Surprisingly, these results were consistent with metastatic adenocarcinoma of the prostate involving the right breast. 
Mandaliya et al.: Prostate Cancer: Cases of Rare Presentation and Rare Metastasis

\section{Discussion}

Metastasis to breast is most commonly seen with contralateral breast cancer and only $2 \%$ of all breast tumours are originating from non-mammary cancers [3]. The most common non-mammary cancers which metastasise to the breast are leukaemia, melanoma, lymphoma, ovarian cancer, lung cancer and gastric cancer [3]. Prostate cancer on the other hand metastasises more commonly to the bones, lung, liver, pleura and adrenal glands [4]. The rare occurrence of metastasis to breast is suggested to be due to the breast having large areas of fibrous tissue with a relatively poor blood supply [5]. Metastasis to the breast is even more unlikely in males, 5-6 times less frequent than in females [6]. There is evidence that suggests that hormonal manipulation in prostate cancer treatment could predispose to breast metastasis. Oestrogen therapy, which was previously the mainstay in the treatment of prostate cancer, has been linked to the development of breast metastasis [2, 7]. Although our patient was not treated with oestrogen therapy, non-steroidal anti-androgen therapy (bicalutamide) is known to increase circulating levels of oestrogen due to competitive inhibition of central androgen receptors which blocks the negative feedback loop therapy increasing luteinizing hormone secreation which leads to increased production of testosterone which becomes aromatised to oestrogen. Despite this, there is no clear predisposing factors found correlating with the development of breast metastasis, and there have been few recorded cases of prostate cancer which metastasised to the breast without any prior hormonal therapy [8]. Other differentials for breast enlargement are gynaecomastia, primary breast cancer or metastasis to the breast from cancer which is rare too. Histopathology from a breast lump including immunohistochemistry is a useful tool to derive a final diagnosis [9]. PSA and PAP (prostatic acid phosphatase) stains on IHC (immunohistochemistry) would certainly help to differentiate the nature of the pathology [10]. Although PSA shows some activity in gynaecomastia, PAP is more specific for metastasis from prostate cancer [11]. Metastatic disease of non-mammary neoplasms involving infiltration of the breast carries a grave prognosis and indicates widely disseminated disease [6].

Brain metastasis is common in cancer, but prostate cancer rarely metastasises to the brain [12]. Approximately $1.6 \%$ of prostate cancer patients have brain metastasis in a large retrospective analysis of 16,280 patients with prostate cancer [13]. Cerebral metastasis from prostate cancer as an initial presentation is extremely rare [14]. The predominant clinical presentation of brain metastasis from prostate carcinoma was confusion and related to intracranial hypertension or diffuse cortical dysfunction such as delirium (50\%), headache (34\%), short-term memory deficits (17\%) and hemiparesis (5\%) [13]. Treatments available for intracranial metastasis include neurosurgery, radiation therapy and hormonal manipulation [15]. Our case presented with predominantly right-sided weakness and underwent neurosurgical resection and radiotherapy followed by androgen deprivation therapy. His disease state is still under control.

\section{Conclusion}

Brian metastasis from prostate cancer is a rare event, and neurological symptoms as a first clinical manifestation are reported in limited cases only. Brain metastasis in prostate cancer should be treated similarly like any other brain metastasis. Similarly, breast metastasis from prostate cancer is a very uncommon entity. To differentiate it from gynaecomastia and metastasis from other source is very much essential, as treatment and prognosis widely varies. 
Mandaliya et al.: Prostate Cancer: Cases of Rare Presentation and Rare Metastasis

\section{Statement of Ethics}

The authors have no ethical conflicts to disclose.

\section{Disclosure Statement}

The authors have no conflicts of interest to declare.

\section{References}

1 Catane R, Kaufman J, West C, et al: Brain metastasis from prostatic carcinoma. Cancer 1976;38:2583-2587.

-2 Hesdorffer C, Bezwoda WR: Metastases of prostate cancer to breast. A case report. S Afr Med J 1987;72:504505.

-3 Njiaju UO, Truica CI: Metastatic prostatic adenocarcinoma mimicking inflammatory breast carcinoma: a case report. Clin Breast Cancer 2010;10:E3-E5.

-4 Bubendorf L, Schopfer A, Wagner U, et al: Metastatic patterns of prostate cancer: an autopsy study of 1,589 patients. Hum Pathol 2000;31:578-583.

5 Mun SH, Ko EY, Han BK, et al: Breast metastases from extramammary malignancies: typical and atypical ultrasound features. Korean J Radiol 2014;15:20-28.

6 Smymiotis V, Theodosopoulos T, Marinis A, et al: Metastatic disease in the breast from nonmammary neoplasms. Eur J Gynaecol Oncol 2005;26:547-550.

7 Moldwin RM, Orihuela E: Breast masses associated with adenocarcinoma of the prostate. Cancer 1989;63:2229-2233.

8 Mostofi FK: Grading of prostatic carcinoma. Cancer Chemother Rep 1975;59:111-117.

-9 Sahoo S, Smith RE, Potz JL, Rosen PP: Metastatic prostatic adenocarcinoma within a primary solid papillary carcinoma of the male breast. Arch Pathol Lab Med 2001;125:1101-1103.

10 Green LK, Klima M: The use of immunohistochemistry in metastatic prostatic adenocarcinoma to the breast. Hum Pathol 1991;22:242-246.

11 Kidwai N, Gong Y, Sun X, et al: Expression of androgen receptor and prostate-specific antigen in male breast carcinoma. Breast Cancer Res 2004;6:R18-R23.

12 Fervenza FC, Wolanskyj AP, Eklund HE, Richardson RL: Brain metastasis: an unusual complication from prostatic adenocarcinoma. Mayo Clin Proc 2000;75:79-82.

13 Tremont-Lukats IW, Bobustuc G, Lagos GK, et al: Brain metastasis from prostate carcinoma: The M D Anderson Cancer Center experience. Cancer 2003;98:363-368.

14 Kasabian NG, Previte SR, Kaloustian HD, Ganem EJ: Adenocarcinoma of the prostate presenting initially as an intracerebral tumor. Cancer 1992;70:2149-2151.

15 Kunkler RB, Cooksey G, Millac P: Carcinoma of the prostate presenting with a cerebral metastasis. Br J Urol 1993;71:103-104. 